\title{
Importance of Sex Education from the Adolescents' Perspective: A Study in Indonesia
}

\author{
Siti Maimunah \\ University of Muhammadiyah Malang, Faculty of Psychology, INDONESIA
}

Received 10 April 2019 • Revised 21 June 2019 • Accepted 18 July 2019

\begin{abstract}
This study aims to explore the adolescents' perspective on sex education as a policy basis of determining whether or not sex education is of importance for adolescents. To date, the concept of sex education is still considered taboo in the community and therefore rarely gets concerned at both home and school. This particularly has an impact on finding less-accurate information among adolescents that leads them into free and risky sexual behaviors. The subjects in this study were 165 adolescents aged 12-19 years. The results of the study show that sex education is of utmost importance from the adolescents' perspective. The material contents of sex education according to respondents' perspective include (1) how to avoid sex and promiscuity; (2) the knowledge improvement on proper sex education; (3) the impacts of free-sex, including sexually transmitted diseases and teenage pregnancy; (4) self-protection (e.g. from free-sex and sexual harassments). This proves the necessity for both parents and teachers to provide adolescents with sex education.
\end{abstract}

Keywords: sex education, perspective, adolescent.

\section{Introduction}

A report from the World Health Organization (WHO) released on 13 December 2018, cites that more than 1.1 million adolescents aged 10-19 years died in 2016 or more than 3,000 of which per day. Most of the causes of their deaths were traffic injuries, HIV/AIDS, labor, and the practice of abortion (Wight, Plummer \& Ross, 2012).

In developing countries, approximately around 16 million girls aged 15-19 years and 2.5 million girls under the age of 16 give birth each year. Generally, the complication during pregnancy and labor emerges as the main cause of their deaths. Annually, nearly 3.9 million girls aged 15-19 years undergo unsafe abortions that ultimately end their lives. An adolescent mother (aged 10-19 years) faces a higher risk of eclampsia, endometritis during puerperium, and of a systemic infection than those of aged 20 years and over. Such higher risk also impacts on adolescents' deaths (WHO, 2018).

The underlying factors of the high adolescents' pregnancy rate, according to Achema, Emmanuel and Moses (2015), incorporates a lack of parental care and guidance (46.7\%), a lack of self-control (36.7\%), and a lack of sex education (13.3\%). It also corresponds to the argument of Papri et al. (2016) stating that the underlying factors of the high pregnancy rate among adolescents

(C) Authors. Terms and conditions of Creative Commons Attribution 4.0 International (CC BY 4.0) apply. Correspondence: Siti Maimunah, University of Muhammadiyah Malang, Faculty of Psychology, INDONESIA. E-mail: sitimaimunah20@gmail.com. 
include low socioeconomic status, low education level, the family disruption, and the substandard sexual health services and practices.

Several studies related to adolescent's sexual behaviors suggest that sex education becomes an alternative to reduce the high rate of sexual activities among adolescents. The rightful authority to provide sex education is both parents and teachers. Parents possess an emotional power to set particular rules during parental care. Therefore, it is their responsibility to provide their children with sex education as they grow up. Meanwhile, a teacher is a respectful model for adolescents and thus appropriate to provide sex education in the school environment. However, in reality many schools have not been ready yet to put sex education into the school curriculum. Many parents are also still reluctant to offer issues related to sex education to their children due to being considered taboo.

Byers et al. (2003) in their research point out that $93 \%$ of students agree that sex education must be offered in the school curriculum and $69 \%$ of which agree that both school and parents are responsible for this. Further, they convey that sex education should be offered in middle school and early. Meanwhile, according to Jimmy et al. (2013), nearly 35\% of students assert that a teacher is the utmost important source of information pertaining to sexual health. This is due to the fact that most of them believe upon what has been taught by their teachers in the classroom. Apart from their parents, students mostly spend their time consulting about right information related to any issues of sexuality and of sexual health. At the same time, 33\% of students believe that it is the parents who should take responsibility for sex education.

Indonesia is a part of Asian countries that remains to emphasize the eastern traditions in varied aspects, one of which is regarding sexual issues. These issues are still considered taboo for most of the Indonesian people; and therefore, it is highly unlikely for adults to talk about such issues with adolescents. By considering all of these, the purpose of this study is, therefore, to explore and access the adolescents' perspective in addressing sex education. This study is expected to be of benefit for consideration whether or not sex education should be offered to adolescents.

\section{Methods}

This study used a qualitative method by means of grounded theory scrutinizing the adolescents' perspective on sex education. Albeit there have been many studies concerning sex education, most of which are, in fact, grounded based on the adults' perspective. The subjects of this study consisted of 165 adolescents.

The collected data were then analyzed by using a coding technique, i.e. categorizing data segments and short names while summarizing and accumulating every data constituents (Charmaz, 2006). This code would show how data were selected, separated, and arranged in order to initiate the data analysis. Principally, coding is an initial phase to a concrete statement in the data interpretation. During the process of coding, three phases suggested by Strauss and Corbin (2007) were used, namely: open coding, axial coding, and selective coding.

Open coding is the process of detailing, examining, comparing, conceptualizing, and categorizing data. Axial coding is the further phase of open coding in which all data were recollected and connected among categories. Last, selective coding is the process of selecting the core categories, connecting them to other categories systematically, and validating the relationship between two. In analyzing the data, the MAXQDA software was used to code word-to-word, sentence-to-sentence, and ultimately paragraph-to-paragraph. 


\section{Findings}

The total subjects filling up the questionnaires were 165 respondents. The demography of respondents' hometown covered Malang, Surabaya, Lamongan, Madiun, Subang, Cianjur, Bogor, Bandung, Indramayu, Balikpapan, and Masohi. Nevertheless, due to the small percentage of each city and mostly dominated by Malang city, it is deemed necessary not to mention the respondents' hometown in the table below.

Table 1. Demographic data of respondents

\begin{tabular}{lcc}
\hline \multicolumn{3}{c}{ Demographic Data of Respondents } \\
\hline \multicolumn{2}{c}{ Total } & Percentage \\
\hline Gender & 51 & $31 \%$ \\
$\quad$ Male & 114 & $69 \%$ \\
$\quad$ Female & & \\
Age & 33 & $20 \%$ \\
$\quad 12-15$ years & 132 & $80 \%$ \\
$\quad 16-19$ years & & \\
Education & 4 & $2.40 \%$ \\
$\quad$ Junior High School & 145 & $88.10 \%$ \\
$\quad$ Senior High School & 16 & $9.50 \%$ \\
$\quad$ University & & \\
In relationship & 108 & $65.50 \%$ \\
$\quad$ Ever & 57 & $34.50 \%$ \\
$\quad$ Never & & \\
Religion & 150 & $91 \%$ \\
$\quad$ Islam & 14 & $8.40 \%$ \\
$\quad$ Protestant & 1 & $0.60 \%$ \\
$\quad$ Catholic & &
\end{tabular}

From the table above, it can be inferred that the majority of respondents are female Muslim senior high school students aged over 15 years who have been in a relationship.

In order to dig up the adolescents' perspective on sex education, the questionnaires comprise two following questions: (1) how important, in your opinion, should sex education be offered to adolescents? and (2) please elaborate the previous questions. From the first question, there were $73.8 \%$ of respondents agreeing that sex education is of importance for adolescents. Meanwhile, there were $21.4 \%$ of respondents stating that it is rather important; and the rest $4.8 \%$ of respondents thought that sex education is not important for them.

Furthermore, the second question pertaining to respondents' rationale upon the importance of sex education indicates that there were 2,739 words included in open coding; afterwards, 128 words of which were selected as the coding. After the scrutiny of both axial and selective coding, there are numerous categories of sex education themes in the adolescents' perspective as presented in this following table: 
Table 2. Sex education in the adolescents' perspective

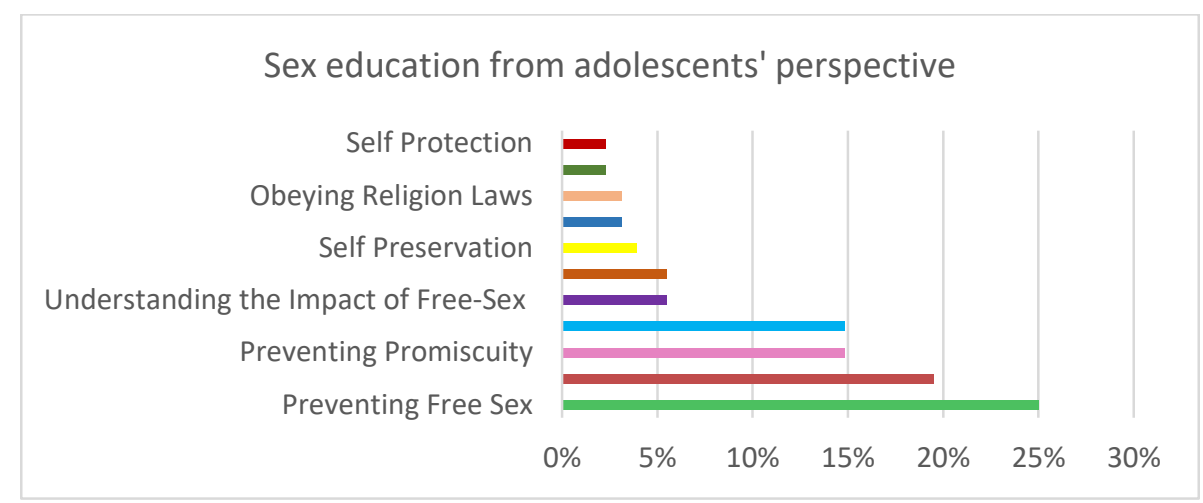

(1) Preventing free-sex

Preventing free-sex becomes the utmost emerging theme revealed by 25\% of respondents. In this theme, the common words used are avoiding, preventing, not falling into, and anticipating. These words appear in several respondents' statements, such as:

"Sex education is important because it is useful in the future and prevents freesex." (Subject 8)

"In order to prevent free-sex in adolescence, it is important to be aware of sex education." (Subject 28)

From the statements above, it can be concluded that from the adolescents' perspective, sex education is essentially important in order to prevent freesex.

(2) Acquiring the proper information on sex education and expanding their knowledge

This theme emerges from the combination between $19.5 \%$ in acquiring the proper information on sex education and $14.8 \%$ in expanding their knowledge. This theme was intentionally combined into a single explanation due to the shared words used by respondents, which are education and knowledge. The endpoint of this theme relies on both propositions that adolescents should get the proper information on sex education in order to expand their knowledge. This can be inferred from these two following statements:

“...since the proper information on sex education in this current era appears to be the most demanded information among adolescents since we know that the current adolescence life is rather free; and knowledge about religion and the risks of sexual behaviours are deemed low." (Subject 84)

"...since in the adolescence, it is important to understand sex education for the sake of future knowledge. Such education can be implemented today or later in the future." (Subject 116)

(3) Preventing promiscuity

This theme emerges from $19.5 \%$ of respondents. The proposition used in this theme is quite clear that sex education should be offered in adolescence in order to prevent promiscuity. Since the exact word used is promiscuity, the use of other words (or synonyms) is deemed unnecessary. These following statements indicate how the adolescents' perspective regarding this theme:

“...since we, as adolescents, who are undergoing the phase of developing our identity, it, therefore, seems reasonable to think that sex education is an essential 
way to prevent promiscuity which likely happens among current adolescents." (Subject 39)

“...since it expands knowledge of nowadays adolescents, who tend to be easily influenced by negative circumstances, such as promiscuity and sex.”(Subject 141)

(4) Understanding the impact of free-sex (e.g. from sexually transmitted diseases and pregnancy)

This theme comprises two sub-themes: sexually transmitted diseases and pregnancy. Separately, the sub-theme of "understanding the impact of free-sex" emerges from 5.5\% of respondents whereas other two sub-themes "preventing sexually transmitted diseases" and "preventing pregnancy" emerge from $5.5 \%$ and $2.3 \%$ respectively. Thus, it can be inferred that this theme reaches $13.3 \%$ of respondents. The theme under "understanding the impact of free-sex" uses the same exact phrase the impact of free-sex (i.e. can be classified into positive impact, negative impact, personal impact, and communal impact). Meanwhile, the two sub-themes use rigid words, such as sexually transmitted diseases, deadly diseases, unwed pregnancy, and teenage pregnancy.

"...therefore, we are able to understand what sex is and its positive and negative impacts on us. The most important thing is that it helps to prevent free-sex, etc." (Subject 126)

"We will get a better understanding of the importance of sex education and thus become more cautious about others' bad influence. At the very least, we can control and understand the impact of free-sex upon ourselves, others, and the community." (Subject 37)

(5) Self-protection (avoiding sexual harassments or abuses)

The theme of self-protection comprises two sub-themes, which are self-preservation (3.9\%) and self-protection (2.3\%). Thus, if combined, it covers $6.2 \%$ of respondents. This theme emerges along with particular expressions such as health protection and future protection. Hence, it can be concluded that the adolescents' perspective on sex education is more likely about how they can protect themselves, their health, their future, and prevent them from sexual harassments or abuses. It can be inferred from these following statements:

“...because sex education must be taught early, let alone for adolescents who seem to neglect its importance. Especially when we talk about self-protection upon other bad people who are willing to harm ourselves in various ways, such as sexual harassments that increasingly happen lately due to lack of knowledge about sex education, of self-protection, and of civilization in the community." (Subject 162)

"...in order to understand the danger of free-sex or premarital sex; in order to understand and protect ourselves from those who are willing to harass." (Subject 164)

(6) Obeying religion laws

In this theme, respondents tend to perceive premarital sex is of religious prohibition. By having a better understanding of sex education, respondents believe that they have obeyed religion laws, specifically by not engaging in free-sex or premarital sex. This theme merely covers $3.1 \%$ of respondents.

“...in order to avoid such things, because in my religion it is strictly prohibited to engage in free-sex or premarital sex (zina)." (Subject 18)

“...because in Islam, it is prohibited to engage in free-sex or premarital sex (zina) as stated in Al-Qur'an." (Subject 20) 


\section{Discussion}

Research findings show that according to respondents, sex education is of importance, by which they are able to avoid/prevent free-sex, promiscuity, sexually transmitted diseases, teenage pregnancy, and to expand their knowledge, understand the proper information on sex education, obey religious laws, as well as self-protection.

Almost all rationales given by respondents relate to the advantages and content materials of sex education for adolescents. The table below illustrates the comparison between respondents' perspective and sex education objectives according to International Planned Parenthood Federation (IPPF, 2010):

Table 3. The comparison of sex education objectives

\begin{tabular}{|l|l|}
\hline \multicolumn{1}{|c|}{ IPPF } & \multicolumn{1}{|c|}{ Adolescents' Perspective } \\
\hline $\begin{array}{l}\text { Obtain accurate information about sexual } \\
\text { and reproductive rights; provide } \\
\text { information about untrue myths; provide } \\
\text { references on sexual health services and } \\
\text { resources. }\end{array}$ & $\begin{array}{l}\text { Obtain accurate information pertaining } \\
\text { to sex education; expand their } \\
\text { knowledge; understand the impacts of } \\
\text { free-sex; avoid/prevent sexually } \\
\text { transmitted diseases and teenage } \\
\text { pregnancy. }\end{array}$ \\
\hline $\begin{array}{l}\text { Develop life skills such as critical thinking, } \\
\text { communication and negotiation skills, self- } \\
\text { development skill, decision-making skill, } \\
\text { self-awareness, self-confidence, } \\
\begin{array}{l}\text { assertiveness, and the ability to ask } \\
\text { questions as well as seek help and empathy. }\end{array}\end{array}$ & Avoid free-sex and promiscuity. \\
\hline $\begin{array}{l}\text { Maintain positive attitudes and values, } \\
\text { including open-mindedness, respect upon } \\
\text { oneself and others, develop positive self- } \\
\text { esteem, conformity, avoid prejudice, be } \\
\text { responsible, and demonstrate positive } \\
\text { attitudes towards sexual health and } \\
\text { reproduction. }\end{array}$ & Obey religious laws and self-protection. \\
\hline
\end{tabular}

From the table above, it can be seen that there are several equal considerations yet being elaborated differently. Referring to the findings, the utmost emerging theme is avoiding/preventing free-sex. This theme can be classified into a single category which shares equal description to the category of decision-making skills in IPPF. In an attempt to avoid/prevent free-sex, surely it cannot be separated from the decision-making skill. Other categories that are related to this include self-awareness and assertiveness. Adolescents will never be able to avoid/prevent free-sex if they do not have high self-awareness about their future and assertiveness to reject any bad influences from surroundings. Avoiding promiscuity also includes in this category. Therefore, it can be concluded that the category of avoiding promiscuity is also equal to the category of decision-making skill in IPPF.

The second category that can be formulated in this study (based on the total occurrences) is obtaining proper information on sex education. By understanding this, adolescents can surely protect themselves by knowing both positive and negative impacts of free-sex and promiscuity, and thus can avoid/prevent sexually transmitted diseases and teenage pregnancy. Referring to IPPF, such category shares equal characteristics to the category of obtaining accurate information about sexual and reproductive rights.

Furthermore, the next categories that have more or less the same occurrence include: obeying religious laws, and self-protection. These categories are equal to self-respect, positive selfesteem, and responsible as the sub-category of maintaining positive attitudes and values in IPPF. 
From the overall comparison of IPPF, there are several categories that do not emerge in the adolescents' perspective pertaining to the impacts of sex education, namely: (1) references to sexual health services and resources; (2) developing life skills including critical thinking, communication and negotiation skills, self-development skills, self-confidence, ability to ask questions and seek help as well as empathy; (3) maintaining positive attitudes and values, including open-mindedness, respect upon oneself and others, develop positive self-esteem, conformity, avoid prejudice, be responsible, and demonstrate positive attitudes towards sexual health and reproduction.

Due to differences and similarities between the IPPF categories and the adolescents' perspective, it can be concluded that adolescents are actually aware of the importance of sex education. Even, most of them (73.8\%) argue about its importance. However, their understanding of sex education material seems very limited; thus, it is highly recommended to arm them with sex education. The materials of sex education supposedly cover these following aspects: (1) how to avoid sex and promiscuity; (2) proper information about sex education; (3) the impacts of freesex; (4) how to avoid sexually transmitted diseases and teenage pregnancy; (5) how to avoid sexual harassments; and (6) how to obey religious laws by avoiding free-sex. For the sake of perfection and suitability to the International Planned Parenthood Federation (IPPF), the materials of sex education can be extended to uncovered aspects that have been discussed in the previous elaboration.

Due to the importance of sex education in the adolescents' perspective, both parents and teachers should not need to feel taboo to provide sex education to adolescents at home and school. By possessing sufficient knowledge on sex education, it is expected that there will be no longer risky sexual behaviors among adolescents so that the negative impacts of free-sex can be prevented or at least minimized. Ergo, one of the main goals in the 203o Agenda for Sustainable Development (SDGs) point 3 and $3 B$ about "ensuring a healthy life and improving people's welfare of all ages" can be achieved.

The limitation of this study covers any yet-developed models of sex education that are suitable for Indonesian cultures and norms, bearing in mind that principally Indonesia still has differences in terms of cultures and norms from European and American countries. Hence, it is recommended for further researchers to conduct in-depth studies on sex education models that are suitable for Indonesian cultures and norms.

\section{Conclusions}

This study presents the importance of sex education from the adolescents' perspective. The concept of sex education, according to adolescents, includes:

(1) How to avoid/prevent free-sex and promiscuity;

(2) How to expand knowledge and to obtain proper information about sex education;

(3) Understand the impacts of free-sex, including sexually transmitted diseases and teenage pregnancy;

(4) Self-protection (i.e. from sexual harassments or abuses);

(5) Obeying religious laws in order to avoid/prevent free-sex.

Due to the importance of sex education from the adolescents' perspective, the policy of offering sex education for adolescents should be reconsidered - even should be changed paradigmatically. Parents and teachers are highly recommended to provide adolescents with sex education at home and at school. 


\section{Acknowledgements}

This research did not receive any specific grant from funding agencies in the public commercial, or not-for-profit sectors.

The author declares no competing interests.

\section{References}

Achema, G., Emmanuel, A., \& Mosses, A. O. (2015). Factors responsible for teenage pregnancy and its implication on adolescent health and education: Perception of secondary school students in Nigeria. International Journal of Medical and Health Research, 1(2), 48-51.

Byers, E. S., Sears, H. A., Voyer, S. D., Thurlow, J. L., Cohen, J. N., \& Weaver, A. D. (2003). An adolescent perspective on sexual health education at school and at home: II middle school student. The Canadian Journal of Human Sexuality, 12(1), 19-33.

Charmaz, K. (2006). Constructing grounded theory: A practical guide through qualitative analysis. London. California. New Delhi. Sage Publication.

International Planned Parenthood Federation / IPPF (2010). IPPF framework for comprehensive sexual education. London. Doortje Braeken, Tim Shand and Upeka de Silva, IPPF Adolescents Team.

Jimmy, E. E., Abeshi, S. E., Kalu, O. O., Uwanede, C. C., \& Dominic, A. O. (2013). Perception of students, teachers and parents toward sexuality education in Calabar South local government area of cross river state, Nigeria. Journal of Sociological Research, 4(2).

Papri, F. S., Khanam, Z., Ara, S., \& Panna, M. B. (2016). Adolescent pregnancy: Risk factors, outcome and prevention. Chattagram Maa-O-Shishu Hospital College Journal, 15(1), 53-56.

Strauss A., \& Corbin, J. (2007). Dasar-dasar penelitian kualitatif (Terjemahan) [Basics of qualitative research (Translation)]. Yogyakarta: Pustaka Pelajar.

Wight, D., Plummer, M., \& Ross D. (2012). The need to promote behaviour change at the cultural level: one factor explaining the limited impact of the MEMA kwa Vijana adolescent sexual health intervention in rural Tanzania. A process evaluation. BMC Public Health, 12: 788, 1-12.

World Health Organization. (2006). Defining sexual health. Defining Sexual Health Report of a Technical Consultation on Sexual Health 28-31 January 2002, Geneva Geneva, (Sexual Health Document Series), 1-35.

World Health Organization (2018). Adolescents: Health risks and solutions. Media centre Fact sheet; 2019. http://www.who.int/mediacentre/factsheets/fs345/en/ (accessed 22 January 2019).

World Health Organization (2018). Adolescent pregnancy. https://www.who.int/news-room/factsheets/detail/adolescent-pregnancy (accessed 23 January 2019). 